\title{
TOOLS IN BRIEF
}

\section{CHEMICAL BIOLOGY}

\section{Chemically probing GPCR-ligand interactions}

$\mathrm{G}$ protein-coupled receptors (GPCRs) are involved in major cellular functions, but these seven-transmembrane-helix proteins are notoriously difficult to study. An important question is how class B GPCRs such as corticotropin releasing factor receptor type 1 (CRF1R) interact with their peptide ligands. Coin et al. used genetically encodable cross-linking amino acids to study how CRF1R binds its ligand on the membrane of a live cell. They introduced the photo-cross-linking amino acid Azi into CRF1R, allowing them to map the ligand-binding site on the receptor. They also determined the position of the ligand in the binding pocket by incorporating the chemical cross-linking amino acid Ffact into CRF1R; Ffact reacts specifically with a cysteine residue in the ligand. The resulting spatial restraints allowed them to build a model for peptide ligand binding to CRF1R.

Coin, I. et al. Cell 155, 1258-1269 (2013).

\section{STEM CELLS}

\section{D expansion of human pluripotent stem cells}

Several potential applications of human pluripotent stem cells (hPSCs), whether in screens, for toxicity testing or in the clinic, are likely to require very large numbers of cells. Scalable culture methods for these cells are therefore desirable. Lei and Schaffer describe a simple three-dimensional (3D) culture method based on a thermoreversible hydrogel for long-term culture of hPSCs. The cells can be seeded at low temperature when the gel is liquid, cultured at $37^{\circ} \mathrm{C}$ as suspended aggregates in the solidified $3 \mathrm{D}$ environment and then harvested again with a simple temperature switch. Upon optimization of multiple experimental conditions, this fully defined system permits stable and unprecedented expansion of hPSCs. Lei, Y. \& Schaffer, D.V. Proc. Natl. Acad. Sci. USA doi:10.1073/pnas.1309408110 (18 November 2013).

\section{GENETICS}

\section{TALENs knock out human microRNAs}

microRNAs are important regulators that degrade mRNAs or prevent their translation and thereby have key roles in development and several diseases. Various methods to inhibit microRNAs exist and allow one to study their function, but Kim et al. took a departure from previous piecemeal approaches and designed tools to efficiently knock out 274 of the 1,600 human microRNA loci. The researchers produced a library of 540 pairs of transcription activator-like effector nucleases (TALENs) to delete genes for important human microRNAs and, as a proof of principle, generated single and double knockout cell lines for miR-141 and miR-200c in only a few weeks. The cells showed the expected upregulation of mRNAs containing motifs of the deleted microRNAs.

Kim, Y.-K. et al. Nat. Struct. Mol. Biol. 20, 1458-1464 (2013).

\section{NEUROSCIENCE}

\section{Seeing the brain through a prism}

An opening in the mouse skull, or cranial window, enables imaging the neurons in the living brain using two-photon microscopy. But using this approach, one can generally only image the most superficial layers of the cortex. To overcome this, Andermann et al. use prisms. They inserted 1-mm-sized glass prisms into the mouse brain, under the cranial window. The hypotenuse of the microprism is coated with aluminum and serves as a mirror that projects the incident light. In this way, the researchers imaged across the vertical plane of the prism and captured neurons across all six layers of the cortex. Having shown previously that the method could be used for acute imaging sessions, Andermann et al. now report that the approach also enables long-term imaging experiments. They use a calcium reporter to simultaneously image the activity of neurons across different cortical layers during locomotion behavior and visual stimulation. Andermann, M.L. et al. Neuron 80, 900-913 (2013). 